\title{
Experimental Evidence for the Role of Cantori as Barriers in a Quantum System
}

\author{
K. M. D. Vant, G. H. Ball, H. Ammann and N. L. Christensen, \\ Department of Physics, University of Auckland, Private Bag 92019, \\ Auckland, New Zealand
}

May 27, 2017

\begin{abstract}
We investigate the effect of cantori on momentum diffusion in a quantum system. Ultracold caesium atoms are subjected to a specifically designed periodically pulsed standing wave. A cantorus separates two chaotic regions of the classical phase space. Diffusion through the cantorus is classically predicted. Quantum diffusion is only significant when the classical phase-space area escaping through the cantorus per period greatly exceeds Planck's constant. Experimental data and a quantum analysis confirm that the cantori act as barriers.
\end{abstract}

\section{Introduction}

The study of nonintegrable Hamiltonian systems has contributed much to the understanding of dynamics in both the classical and quantum domains. Classical phase space can contain the rich structure of resonances, Kolmogorov-Arnol'd-Moser (KAM) tori, cantori and regions of stochasticity. Knowing quantum mechanics to be the correct description of physical phenomena has motivated physicists to search for the signatures of these classical structures within the quantum regime. Hypersensitivity to initial conditions, a hallmark of classical chaos, is noticeably absent with quantum mechanics. The study [1-2] and experimental observation of dynamical localization [34] dramatically displayed how quantum mechanics can eliminate stochastic diffusion. There has always been the hope that a clear transition between quantum and classical physics could be achieved, but whereas one can always theoretically allow $\hbar \rightarrow 0$ and realize classical behavior, $\hbar$ 's small but nonzero value always exists in the laboratory. With $\hbar \neq 0$ there is a clear 
distinction between the properties of classical chaos and its associated quantum counterpart.

The presence of KAM tori and cantori in the classical phase space are predicted to influence the corresponding quantum system. If a KAM boundary is unbroken it will prohibit classical diffusion through it, but quantum mechanical tunneling across the barrier is possible. When interaction terms in the perturbing Hamiltonian are sufficiently large so as to break up the boundary and create a cantorus or turnstile, classical particles will quickly diffuse through that cantorus but the quantum wavefunction will be inhibited [5-8]. Given a periodic perturbing Hamiltonian, a heuristic model proposes that when the classical phase-space area escaping through the cantorus each period is $\sim \hbar$ then quantum diffusion is constrained [7-8]. Even though the barrier has been broken, the quantum wavefunction still appears to tunnel through the cantorus. The quantum system somehow senses the cantorus, resulting in a diminished probability for penetration of the barrier than that predicted classically. The structure of this paper is as follows. In Section 2 we introduce the experimental details of our study of cantori constraining the diffusion of quantum particles. The results of our experiment, along with our classical and quantum calculations, are presented in Section 3. We examine the difference between dynamical localization and cantori localization in Section 1 . Finally, a summary is contained in Section 5 .

\section{$2 \quad$ Experimental Details}

A unique and pristine environment for studying quantum chaos is achieved through the use of laser cooled neutral atoms within a modulated standing wave of light [3-4,9-11]. The observation of dynamical localization in the atomic optics realization of the $\delta$-kicked rotor (DKR) [9-11] provided an important experimental link to the most studied system in Hamiltonian chaos. In this present paper we again subject our ultracold caesium atoms to a pulsed standing wave of near resonant light. A periodic pulsed potential of finite width (as used in the DKR experiments [9-11]) produces a KAM boundary, which becomes more noticeable for longer pulse widths. However, a train of single pulses is not the best system for observing diffusion through cantori as the classical phase space outside the boundary is not strongly chaotic and contains many regular regions. We therefore subjected our atoms to a train of double pulses. The pulse train consists of two rectangular pulses of duration $\tau_{p}=1.25 \mathrm{~ms}$ with their leading edges separated by 
$\tau_{s}=2.5 \mathrm{~ms}$. The double pulses occur at every $T=25 \mathrm{~ms}$. Fig. 1(a) displays our double pulse train. Adopting the notation used in [9-11], we can write the dimensionless form of the Hamiltonian as,

$$
H=\frac{\rho^{2}}{2}-k \cos \phi \sum_{l=1}^{N} f(t-l)
$$

where the $f(t)$ specifies the temporal shape of the pulses. For an infinite train of kicks, the Hamiltonian can be written as,

$$
H=\frac{\rho^{2}}{2}-k \sum_{r=-\infty}^{\infty} a_{r} \cos (\phi-2 \pi r t)
$$

where $a_{0}=1 / 10$ and $a_{r \neq 0}=[\sin (3 r \pi / 20)-\sin (r \pi / 20)] /(r \pi)$. The classical interpretation of Eqn. (2) shows that the fundamental resonances are located at $\rho=2 \pi r$. The benefit of using this type of pulsed system is illustrated in Fig. 1(b) where the magnitudes of the $a_{r}$ terms are displayed. The KAM boundary, located at $\rho=10 \pi$ corresponds to the zero energy of the $r=5$ term. Note that there is a relatively large amount of energy for $r>5$. One can see in the Poincare sections displayed in Fig. 2 that the $\rho=10 \pi$ cantorus separates two strongly chaotic regions; the boundary is clearly visible for $k=70$ but is broken up into a chain of tiny islands beyond the resolution of the simulation when $k=300$. Another KAM boundary at $\rho=30 \pi$ provides the upper boundary to the second region, and remains unbroken for all kick strengths used in our experiment. We prepare our atoms so that they initially lie within the $\rho=10 \pi$ cantori, and we monitor their subsequent evolution through the barrier.

In order to establish the connection between the dimensionless parameters used above and those that can be achieved in a laboratory, we consider an atom (transition frequency $\omega_{0}$ ) suspended in a standing wave of near resonant light (frequency $\omega_{l}$ ). Under the assumption of a large detuning compared to the Rabi frequency, the atoms' excited-state amplitude can be adiabatically eliminated. The resulting Hamiltonian governing the coherent time evolution reads [9-11]

$$
H=\frac{p^{2}}{2 m}-\frac{\hbar \Omega_{e f f}}{8} \cos \left(2 k_{l} x\right) \sum_{q=1}^{N} f(t-q T)
$$

where $\Omega_{\text {eff }}=\Omega^{2}\left(s_{45} / \delta_{45}+s_{44} / \delta_{44}+s_{43} / \delta_{43}\right)$ and $\Omega / 2$ is the resonant Rabi frequency corresponding to a single beam. The terms in brackets take account of the different dipole transitions between the relevant hyperfine levels 
in caesium $\left(F=4 \rightarrow F^{\prime}=5,4,3\right)$. The $\delta_{4 j}$ are the corresponding detunings and, assuming equal populations of the Zeeman sublevels, the numerical values for the $s_{4 j}$ are $s_{45}=11 / 27, s_{44}=7 / 36, s_{43}=7 / 108$. The function $f(t)$ represents the shape of our double kicks, discussed above. The dimensionless Hamiltonian is then recovered with $\phi=2 k_{l} x, \rho=2 k_{l} T p / m$, $t^{\prime}=t / T$ and $H^{\prime}=\left(2 k_{l}^{2} T^{2} / m\right) H$; the primes are subsequently dropped. The kick strength is $k=\Omega_{e f f} \omega R T^{2}$, and $\omega_{R}=\hbar k_{l}^{2} / 2 m$ is the recoil frequency. The quantum features of the DKR enter through the commutation relation $[\phi, \rho]=i \hbar$, where $\hbar=8 \omega_{R} T$. The relationship between momenta is $n=p /\left(2 \hbar k_{l}\right)=\rho / \hbar$.

Explicit details of the experimental set-up can be found in [11]. Approximately $10^{5} \mathrm{Cs}$ atoms are initially trapped and cooled in a magneto-optic trap (MOT) to a temperature of $10 \mu \mathrm{K}$. The position distribution of the trapped atoms has a FWHM of $200 \mu \mathrm{m}$. After trapping and cooling the magnetic field gradient, the trapping beam and the repumping beam are turned off, leaving the atoms in the $F=4$ ground state. The atoms are then subjected to a modulated periodic potential from a third laser diode. This beam passes through an acousto-optic modulator (AOM), is collimated to a measured waist $\left(1 / e^{2}\right.$ intensity radius) of $0.95 \mathrm{~mm}$, and is then retroreflected from a mirror outside the vacuum cell to generate the one-dimensional potential. The maximum Rabi frequency at the MOT center is $\Omega / 2 \pi=100 \mathrm{MHz}$, for a total power of $10 \mathrm{~mW}$. The finite widths of the kicking beam waist and the atomic cloud result in a narrow distribution of $k$ with rms spread of $6 \%$ and $k_{\text {mean }} \approx 0.94 k_{\max }\left(k_{\max }\right.$ the value on the beam axis). In the following, when specifying $k$, this always refers to $k_{\text {mean }}$. The kick strength $k$ was varied by adjusting $\Omega$ while maintaining a detuning of $\delta_{45} / 2 \pi=2.8 \mathrm{GHz}$ to the blue. For our detuning the spread in coupling strengths created by differing AC Stark shifts of the different magnetic sublevels was below 1\%. To measure the atomic momentum distribution we use a time-of-flight technique with a 'freezing molasses' [9-11] with an expansion time of $12 \mathrm{~ms}$.

\section{Results from calcualtions and the experiment}

The purpose of the experiment discussed in this paper is to examine the diffusion of particles through the barriers at $\rho= \pm 10 \pi$. Initially our Cs atoms are prepared in the MOT with a temperature of $10 \mu \mathrm{K}$, or a variance of $\sigma_{\rho}=9.2$ in terms of the dimensionless momentum $\rho$. This places the initial momentum distribution within the cantori. After a number of kick cycles of sufficient strength the resulting atomic momentum distribution 
quickly resembles that displayed in Fig. 3. The effects of the cantori are clearly discernible. For this example we had $k=310$ and $N=56$. Note the wings in the distribution outside of the $\rho= \pm 10 \pi$ cantori, but before the $\rho=30 \pi$ boundary. For comparison we display the predicted distributions for quantum and classical calculations. The asymmetry in the measured lineshape around $\rho=0$ is due to fringes in the freezing molasses. The lineshapes are consistent in appearance over two or three days, at which point the entire optical system is typically re-optimized. Fig. 4 displays the temporal evolution of the lineshapes for $k=300$. The traces correspond to $N=3,6,44$ and 68 . There is an initial spreading of the lineshape, followed by a subsequent saturation with shoulders appearing on the lineshape at the location of the $\rho= \pm 10 \pi$ cantori.

In keeping with the work of Geisel et al [5-6] we measured and calculated the percentage of particles that would cross the $\rho= \pm 10 \pi$ cantori as a function of kick number and kick strength. All our measurements were at a fixed "Planck's constant" of $\hbar=2.6$; the consequences of a variation of $\hbar$ in this double pulse system will be the subject of future work. In this present work we also purposely avoid the influence of spontaneous emission. The study of how decoherence introduced via spontaneous emission can affect the ability of particles to flow through the cantori is intriguing, and will be presented in a forthcoming publication. In this present study we accounted for spontaneous emission by including it in our quantum computation, and comparing it to a computation where the effect is absent. We can therefore confirm that our experimental results are from a regime where the influence of spontaneous emission is minimal, but still noticeable. Our quantum analysis is based on a density matrix calculation. Spontaneous emission is included via the inclusion of an interaction term, $H_{i n t}=-\zeta u \hbar \phi \sum_{l=1}^{N} \delta(t-l)$ to the Hamiltonian (1), where $\zeta$ is either 0 or $1,\langle\zeta\rangle=\eta=$ probability for spontaneous emission per double kick, and $u \hbar$ is the recoil momentum projected onto the kicking beam axis ( $u$ chosen randomly on the interval $[-1,1]$ ).

The influence of KAM boundaries on the momentum distribution of atoms in similar experiments has been previously observed [4]. The Austin group has also recently carried out a systematic investigation of the finitetime pulsed rotor system [12], with single kicks. By increasing the duration of the pulses they bring in the KAM boundary and observe a reduction in the energy of the atomic distribution.

There exists a heuristic explanation for the inhibition of quantum diffusion through partial barriers and turnstiles [7-8]. When the flux per period of phase space area escaping through classical cantorus is comparable to or less than Planck's constant, then quantum diffusion is suppressed and pene- 
tration through the barrier is only via tunneling. Our experimental observations and theoretical analysis support this hypothesis. In Fig. 5 we display the results of quantum and classical analyses that predict the percentage of atoms that will be found outside the $\rho= \pm 10 \pi$ cantori as a function of kick number for various kick strengths; both the classical and quantum calculations omit spontaneous emission. The initial distribution corresponds to $10 \mu \mathrm{K}$ or a variance of $\sigma_{\rho}=9.2$, or $99.9 \%$ of the atoms within the $\rho= \pm 10 \pi$ cantori. Our computer simulations reveal that the KAM boundary is broken at $k \leq 50$. With the barrier broken the classical particles will eventually spread themselves uniformly between the $\rho= \pm 30 \pi$ barriers, thereby giving a probability to be found outside the $\rho= \pm 10 \pi$ cantori of $2 / 3$. For $k=310$ the classical particles reach this equilibrium in about 70 kicks.

We have numerically calculated the classical phase space flux through the cantorus per kicking cycle. We assume the particles are uniformly spatially distributed, but with initial momentum of $\rho=10 \pi$. Referring to the kick strengths displayed in Fig. 5, we find that the phase space area per period moving across the cantorus to larger momenta is proportional to $k^{2}$, with values of $5.4 k, 3.4 k$ and $0.88 \hbar$ for $k=310,240$, and 120 respectively. The ability of the cantori to constrain the quantum particles is clearly displayed. The quantum system quickly reaches its own equilibrium, with significantly fewer atoms outside the cantori. Only for kick strengths around $k=1200$ does the quantum system begin to mimic the classical, with the probability for penetration of the cantori exceeding $60 \%$ and the phase space area per period moving across the cantorus at $31 k$.

The experimental results for our diffusion experiment are shown in Fig. 6. Displayed are the measured probabilities of finding our Cs atoms outside the $\rho= \pm 10 \pi$ cantori. Also shown are the results of quantum analyses that include the spontaneous emission rates for the experimental parameters. The spontaneous emission rate per kicking cycle is $\eta=0.021,0.017$ and 0.008 for $k=310,240$ and 120 respectively. We see good agreement between the measured and calculated probabilities. For strong kick strengths the final measured probabilities are noticeably smaller than those predicted classically; for $N \approx 70$ we measure $37 \%$ and $12 \%$ for $k=310$ and 240 respectively, whereas the classical prediction gives $62 \%$ and $48 \%$. The broken cantori still dramatically restrict the movement of atoms through the classically broken barrier. By blocking our retroreflected beam we experimentally confirm that our small spontaneous emission rates create minimal heating and do not produce any movement of the atoms through the cantori.

The resolution of our CCD (19 pixels/mm), coupled with our $12 \mathrm{~ms}$ expansion time, allowed us to determine the position of the momentum line 
to an accuracy of $\Delta \rho= \pm 0.8$. At our strongest kick strength of $k=310$ this results in an uncertainty in our measured probability of $\pm 4 \%$. We repeat our experiment a number of times during each experimental session, and the resulting spread in our measured values is $\pm 4 \%$.

\section{Cantori localization and dynamical localization}

Different process can contribute to a quantum system's inhibition to diffusion. Dynamical localization is certainly the reason for the elimination of momentum diffusion in the kicked rotor [1-2] and its atomic optics realization [9-11] at sufficiently high kick strengths; we note that others [13] disagree with this conclusion for the experiment described in Refs. [3-4]. Recent theoretical work on discontinuous quantum systems displays dynamical localization and cantori inhibition of diffusion in the same system [14]. Previously other studies also focused on the transition from cantori localization to dynamical localization [15]. For the experiment described in this present paper the cantori also act as barriers. Whereas dynamical localization results in an exponential lineshape for the momentum distribution in the $\delta$-kicked rotor, the quantum version of our double kick system quickly settles into the type of distribution that is displayed in Figs. 3 and 4. Our measurements and quantum analysis show that the system's probability for penetration of the barrier saturates at values far below the classical prediction.

The effect of dynamical localization is universal, and appears in a broad class of systems. To some extent it will also contribute to the restriction of momentum diffusion in our double kick system. In order to more closely examine dynamical and cantori localization we compare the double kicked system to one with a single kick, but with the same total pulse area. In the following, the kick strength is $k=300$, while the single kick has a pulse length of $\tau=2.5 \mu \mathrm{s}$, and pulse period $T=25 \mu \mathrm{s}$. For the single kick system there will be no $\rho= \pm 10 \pi$ cantori, but KAM boundaries at $\rho= \pm 20 \pi$. Fig. 7 displays the results of a quantum calculation for the momentum lineshapes after 50 kick cycles. The calculation includes the slight effect of spontaneous emission rate of $\eta=0.02$ per kick cycle for a detuning of $2.8 \mathrm{GHz}$. The single kick lineshape is not perfectly exponential in shape, and is definitely constrained by its $\rho= \pm 20 \pi$ KAM boundary. Similar lineshapes for the finite pulse kicked rotor have been previously experimentally studied [12]. One can clearly observe the different shape for the double kick momentum distribution; the shoulders develop at the location of the $\rho= \pm 10 \pi$ cantori. In Fig. 8 we have the experimentally observed momentum lineshapes for 
the single and double kicked atoms. Once again the shoulders are observed for the double kicked system at the $\rho= \pm 10 \pi$ cantori. The single kick momentum distribution is broad enough such that its localization length would extend to the location of the cantori at $\rho= \pm 10 \pi$. Hence the effect of these cantori become important and noticeable in the double kick system.

If we model our kicked system as a kicked rotor then a prediction can be made for the localization length one would expect from dynamical localization. A kick strength of $k=300$, a single kick pulse length of $\tau=2.5 \mu \mathrm{s}$, and pulse period $T=25 \mu$ s provides a classical stochasticity parameter for the kicked rotor of $\kappa=30$ [10-11]. Dynamical localization would predict a characteristic exponential distribution $\sim \exp \left(-2|\rho| / l_{\rho}\right)$, with localization length $l_{\rho} \approx \kappa^{2} / 2 \hbar \approx 170$ [1]. This greatly exceeds the cantori at $\rho= \pm 10 \pi$ for the double kicked system, hence the shoulders that develop are evidence for cantori localization. For the single kicked system one can observe a boxlike distribution due to the $\rho= \pm 20 \pi$ boundaries, which are also smaller than the localization length.

For our parameters $(k=300, N=50, \delta=2.8 \mathrm{GHz})$ the single kick system saturates to a momentum distribution with an additional $10 \%$ of the total number of atoms in excess of $\rho= \pm 10 \pi$. Fig. 9 compares the percentage of atoms that cross the momentum value of $\rho= \pm 10 \pi$ for the single and double kicks. This calculation includes the spontaneous emission rate of $\eta=0.02$ per kick cycle. Also, the single kick system has a 'quantum break time' of approximately $N=7$, whereas the double kicked system needs about 15 kicks to come to equilibrium. The $\rho= \pm 10 \pi$ cantori slow the initial diffusion of atoms. The quick equilibrium for similar finite length single pulses has been experimentally observed [12], while the slow initial diffusion is clearly seen in our Fig. 6.

\section{Discussion}

Decoherence in the atomic optics manifestation of the kicked rotor has recently been observed [10-11,16]. The decohering effect of spontaneous emission is small but still noticeable in our present data. Whereas a fully quantum system saturates and the percentage of atoms found beyond the $\rho= \pm 10 \pi$ cantori remains constant, spontaneous emission creates a slow drift in the system toward the classical equilibrium configuration where $2 / 3$ of the particles are outside these cantori. The coupling of a quantum system to extraneous degrees of freedom, or the environment, destroys quantum coherences. In these quantum chaos experiments utilizing atomic optics the 
quantum dynamics become susceptible to the decohering effects of spontaneous emission. The environment in this case is the vacuum fluctuations. A heuristic model for decoherence observed in our double kick system, along with further experimental data, will be presented in a forthcoming publication. The model is similar to that presented in [10-11]. However, from an experimental view of our present data one can clearly observe the slow drift of our quantum system towards the classical equilibrium configuration.

By using a periodic train of double (finite width) pulses we create a system with two strongly chaotic regions separated by a cantorus. Using laser cooled atoms and a pulsed laser beam we have a pristine environment for experimentally observing a quantum system's inhibition to diffusion through cantori. Previous theoretical work [5-8] supports the hypothesis that when the flux of classical phase space through turnstiles is less than Planck's constant then the movement of particles across the barrier is restricted. Our measurements and calculations of the movement of atoms through the cantorus support the conclusion that when the classical phase space flux through the cantori per kicking cycle is $\lesssim \hbar$, the system fails to see the holes in the cantori [14]. The restriction of the quantum particle's momentum is remarkable when one considers the underlying classical phase space. For the kick strengths used in our experiment the $\rho= \pm 10 \pi$ cantori are not visible in the Poincare section, just an apparent region of stochasticity constrained by the barriers at $\rho= \pm 30 \pi$. However, the effect of the cantori remains significant for the quantum system.

\section{Acknowledgements}

This work was supported by the Royal Society of New Zealand Marsden Fund and the University of Auckland Research Committee.

\section{References}

[1] D.L. Shepelyansky, Physica D 28, 103 (1987)

[2] S. Fishman, D.R. Grampel, and R.E. Prange, Phys. Rev. Lett. 49, 509 (1982)

[3] F.L. Moore, J.C. Robinson, C. Bharucha, P.E. Williams, and M.G. Raizen, Phys. Rev. Lett. 73, 2974 (1994)

[4] J.C. Robinson, C. Bharucha, F.L. Moore, R. Jahnke, G.A. Georgakis, Q. Niu, M.G. Raizen, and B. Sundaram, Phys. Rev. Lett. 74, 3963 (1995)

[5] T. Geisel, G. Radons, and J. Rubner, Phys. Rev. Lett. 57, 2883 (1986) 
[6] T. Geisel and G. Radons, Physica Scripta 40, 340 (1989)

[7] R.C. Brown and R.E. Wyatt, Phys. Rev. Lett. 57, 1 (1986)

[8] R.S. MacKay and J.D. Meiss, Phys. Rev A 37, 4702 (1988)

[9] F.L. Moore, J.C. Robinson, C.F. Bharucha, B. Sundaram, and M.G.

Raizen, Phys. Rev. Lett. 75, 4598 (1995)

[10] H. Ammann, R. Gray, I. Shvarchuck and N. Christensen, Phys. Rev. Lett. 80, 4111 (1998)

[11] H. Ammann, R. Gray, N. Christensen, and I. Shvarchuck, J. Phys. B: At. Mol. Opt. Phys. 31, 2449 (1998)

[12] B.G. Klappauf, W.H. Oskay, D.A. Steck, and M.G. Raizen, Physica D, in press (1998)

[13] M. Latka and B.J. West, Phys. Rev. Lett. 75, 4202 (1995), and resulting comments, M.G. Raizen, B. Sundaram, and Q. Niu, Phys. Rev. Lett. 78, 1194 (1997), S. Meneghini, P.J. Bardroff, E. Mayr, and W.P. Schleich, Phys. Rev. Lett. 78, 1195 (1997), M. Latka and B.J. West, Phys. Rev. Lett. 78, 1196 (1997)

[14] F. Borgonovi, Phys. Rev. Lett. 80, 4653 (1998)

[15] G. Radons, T. Geisel, and J. Rubner, Adv. Chem. Phys. 73, 891 (1989)

[16] B.G. Klappauf, W.H. Oskay, D.A. Steck, and M.G. Raizen, Phys. Rev. Lett. 81, 1203 (1998) 
(a)

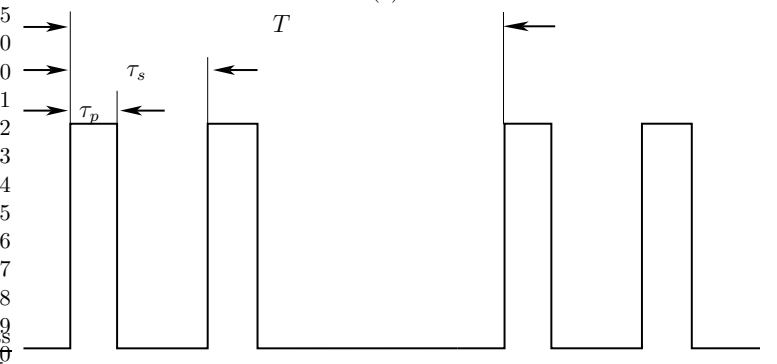

(b)

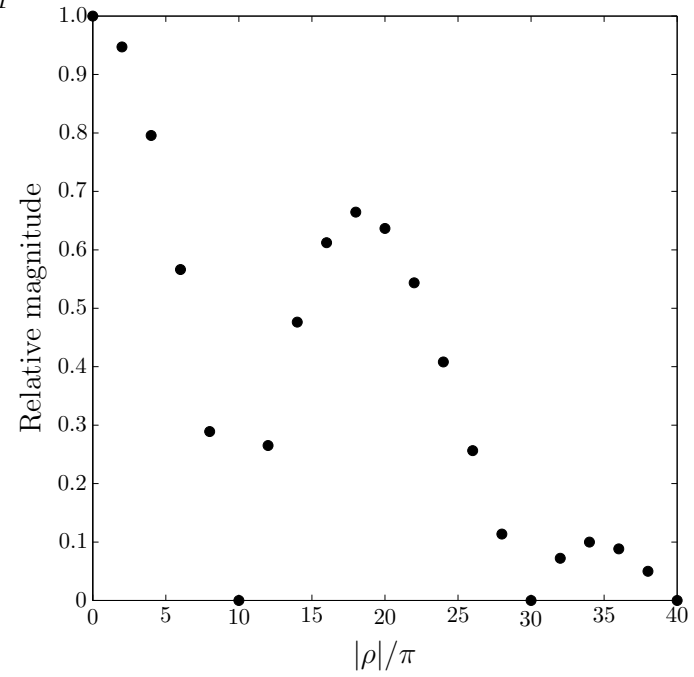

Figure 1: Time sequence (a) of the pulse, and the magnitude of the Fourier components $a_{r}$ (b) used in the Hamiltonian, Eqn. (2). 
(a)

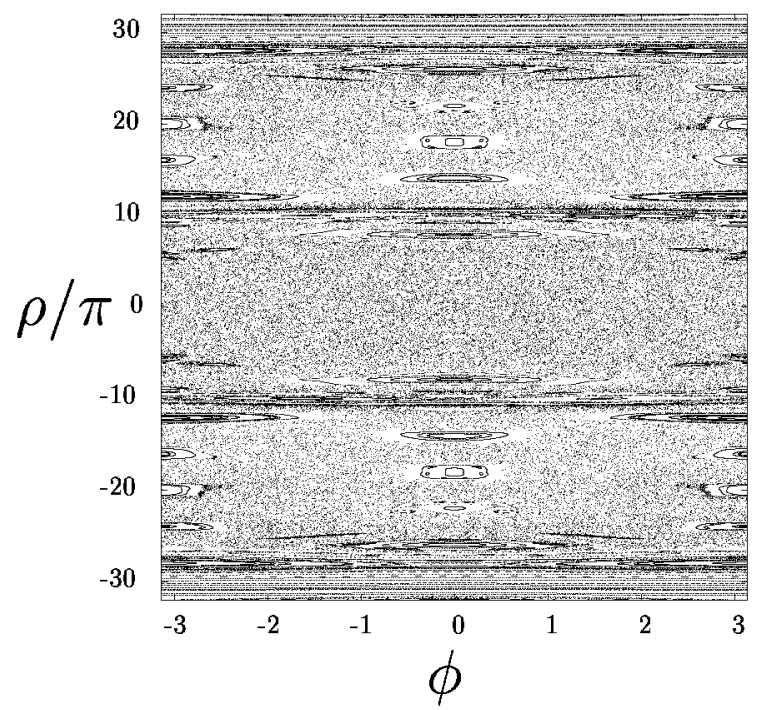

(b)

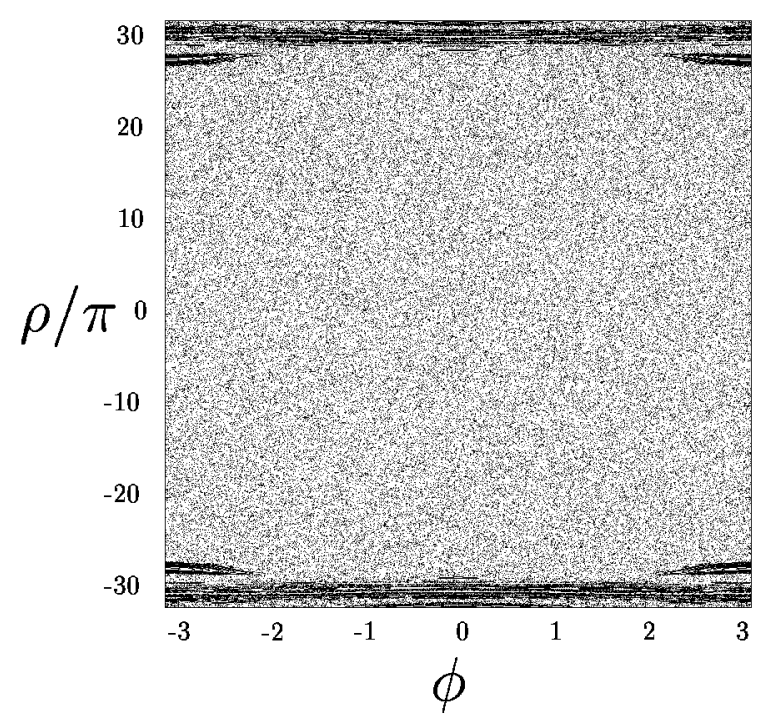

Figure 2: Classical phase space for the relatively small kick strength $k=70$ (a), with boundaries clearly visible at $\rho= \pm 10 \pi$ and $\rho= \pm 30 \pi$. For the kick strength of $k=300$ (b) the $\rho= \pm 10 \pi$ cantori have completely broken up. 


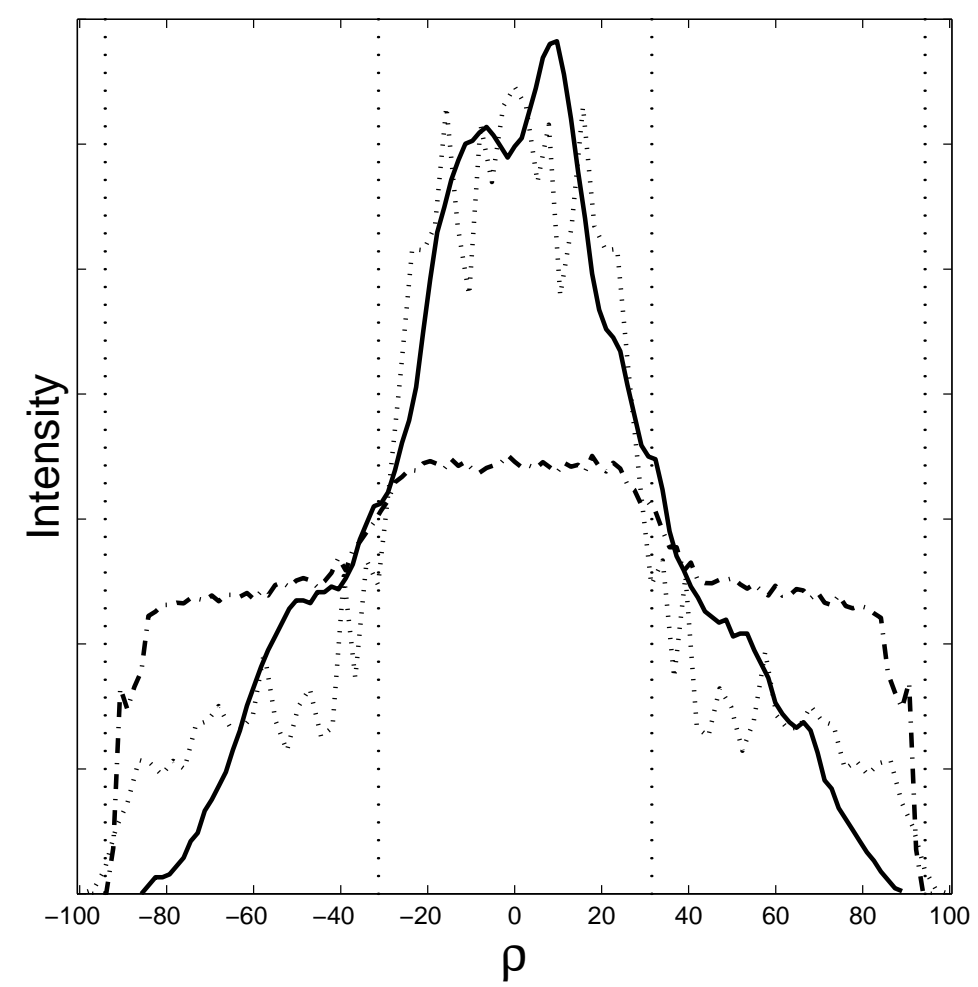

Figure 3: An example of a measured momentum distribution (solid) for kick strength $k=310$ and number of kicks $N=56$, along with that predicted via a quantum (dotted) or classical (dot-dashed) analysis. The vertical lines correspond to the location of the barriers at $\rho= \pm 10 \pi$ and $\rho= \pm 30 \pi$. 


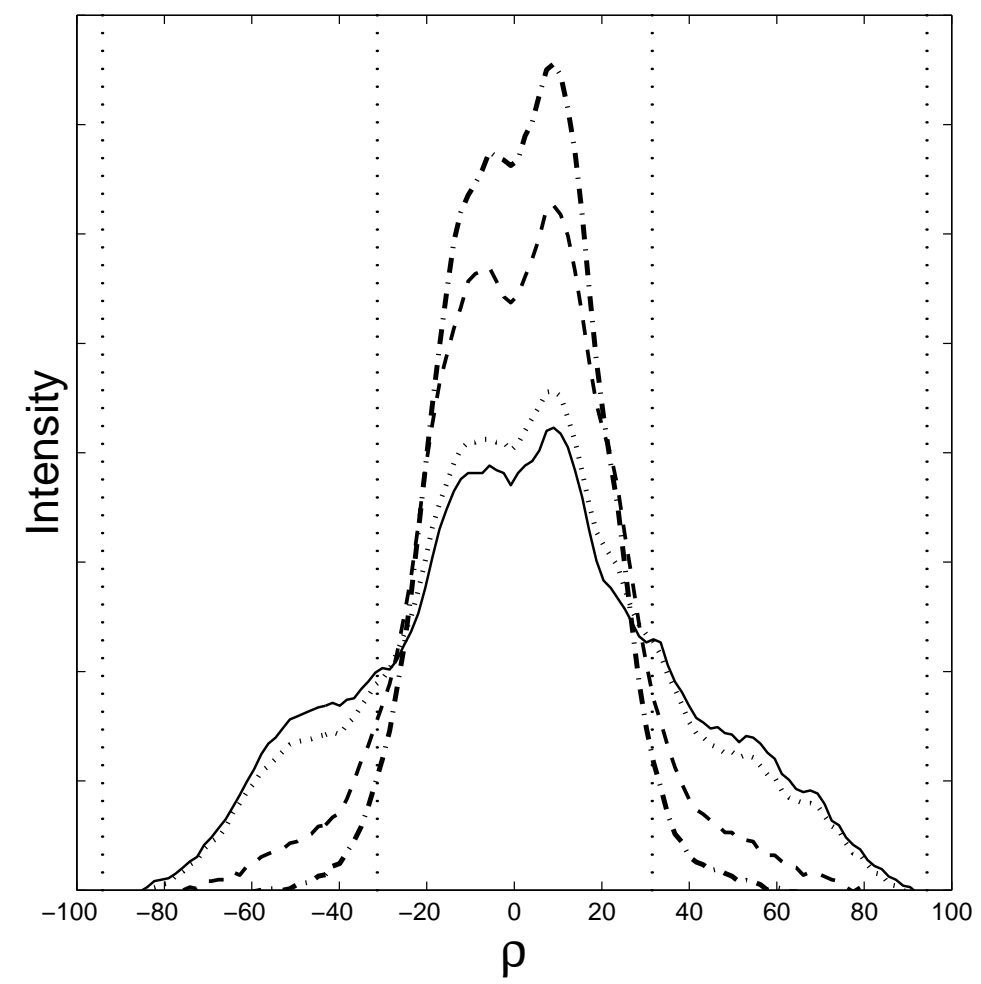

Figure 4: Measured momentum distribution for kick strength $k=300$ as the number of kicks increases; $N=3$ (dot-dashed line), $N=6$ (dashed line), $N=44$ (dotted line), and $N=66$ (solid line). The vertical lines correspond to the location of the barriers at $\rho= \pm 10 \pi$ and $\rho= \pm 30 \pi$. 


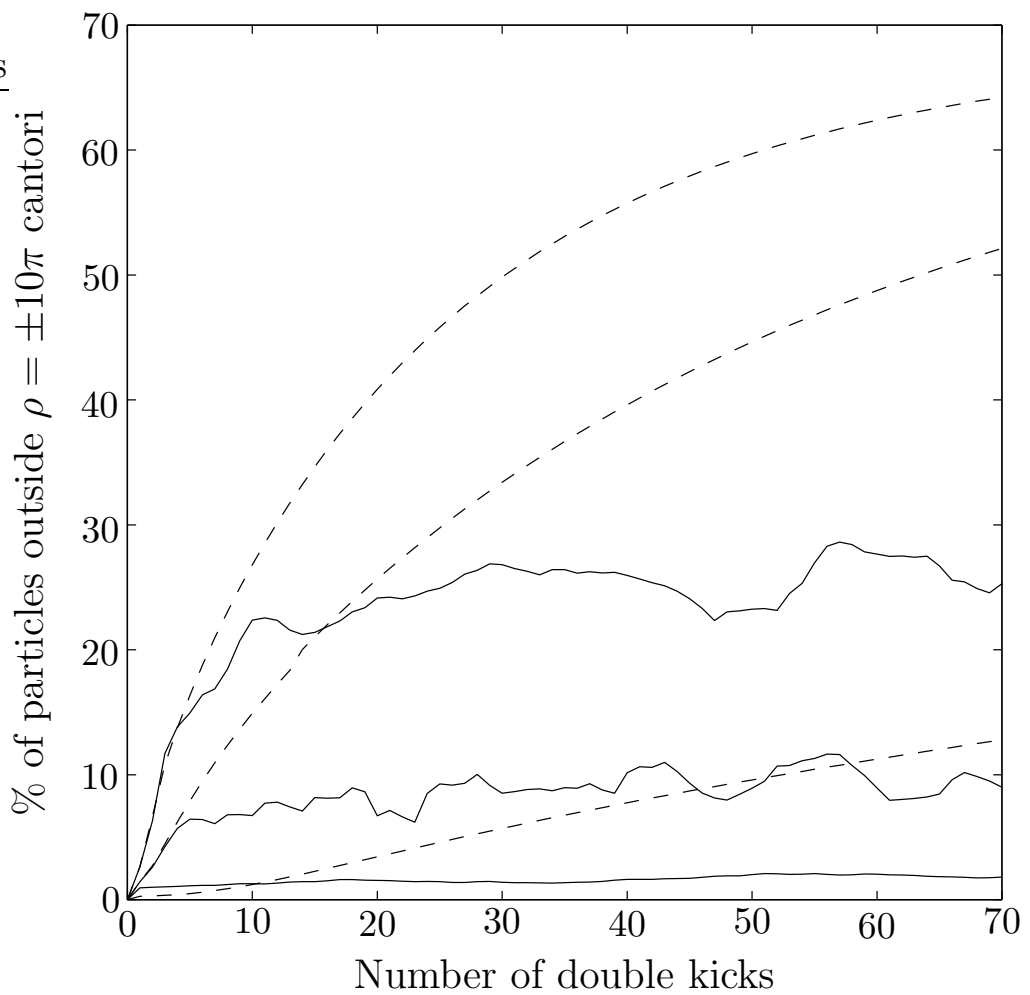

Figure 5: The probability for finding the particles outside the $\rho= \pm 10 \pi$ cantori, versus kick number as calculated via classical (dashed) and quantum (solid) analyses for kick strengths of $k=310$ (upper), 240 (middle), and 120 (lower). 


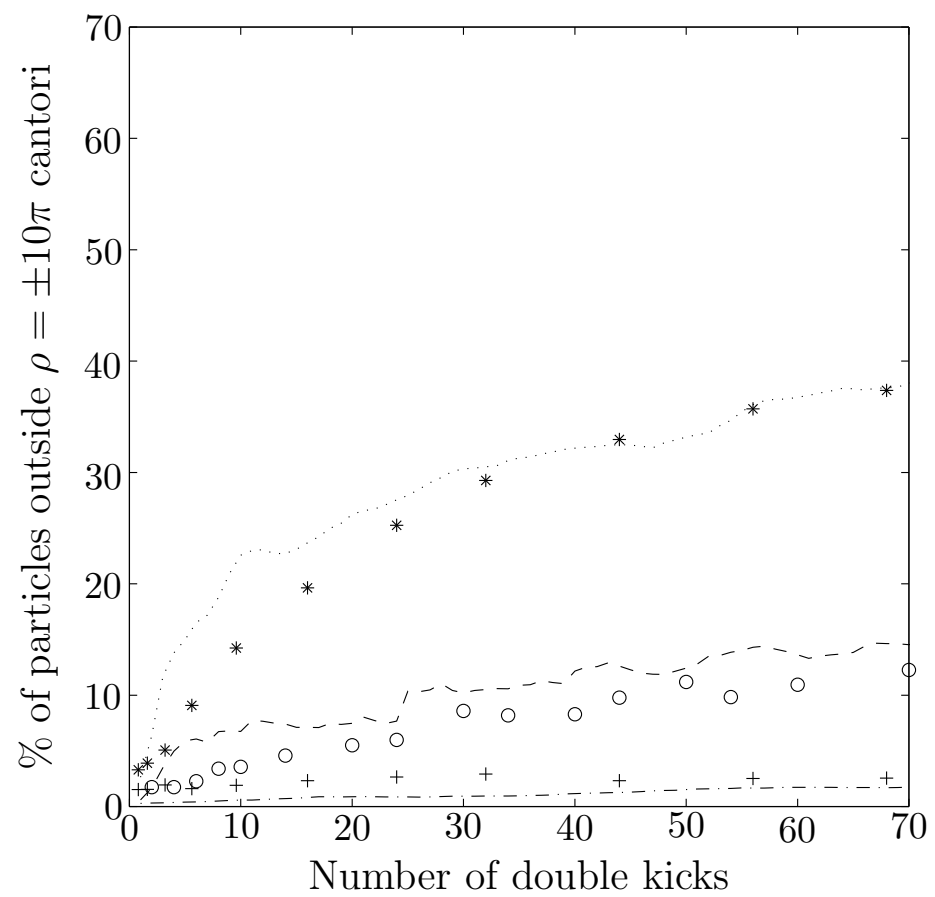

Figure 6: The probability for finding the particles outside the $\rho= \pm 10 \pi$ cantori, versus kick number for experimental (data points) and a quantum simulation (lines) that includes spontaneous emission for kick strengths of $k=310$ ( $^{*}$ and dotted line), 240 ( and dashed line) and 120 ( + and dotdashed line). The position of the $\rho= \pm 10 \pi$ momentum line is determined to an accuracy of $\Delta \rho= \pm 0.8$, resulting in an uncertainty in our measured probability of $\pm 4 \%$. Multiple repetition of our experiment provides a spread in the measured values of $\pm 4 \%$. 


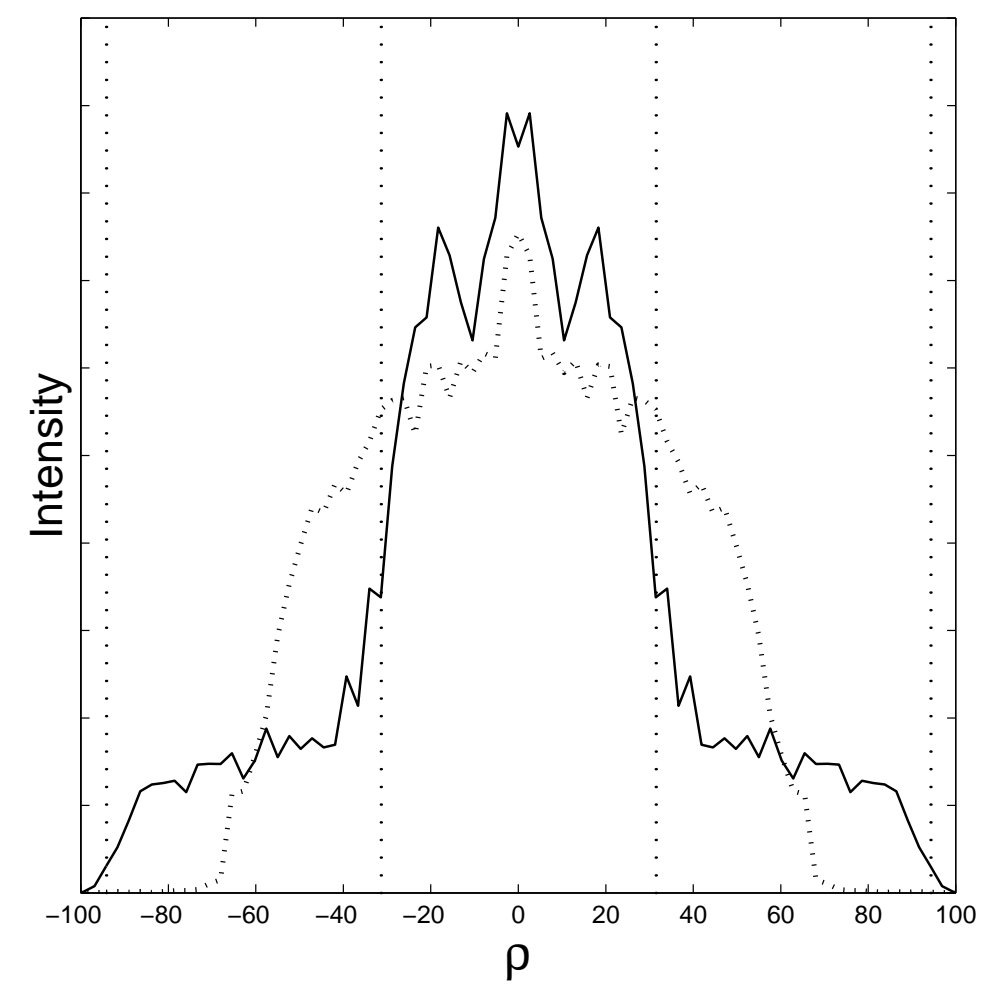

Figure 7: An example of the calculated momentum distribution for kick strength $k=300$ and number of kicks $N=50$ for the double (solid line) and single (dotted line) kicked systems. The vertical lines correspond to the location of the barriers at $\rho= \pm 10 \pi$ and $\rho= \pm 30 \pi$. The calculation includes a spontaneous emission rate of $\eta=0.02$ per pulse period. 


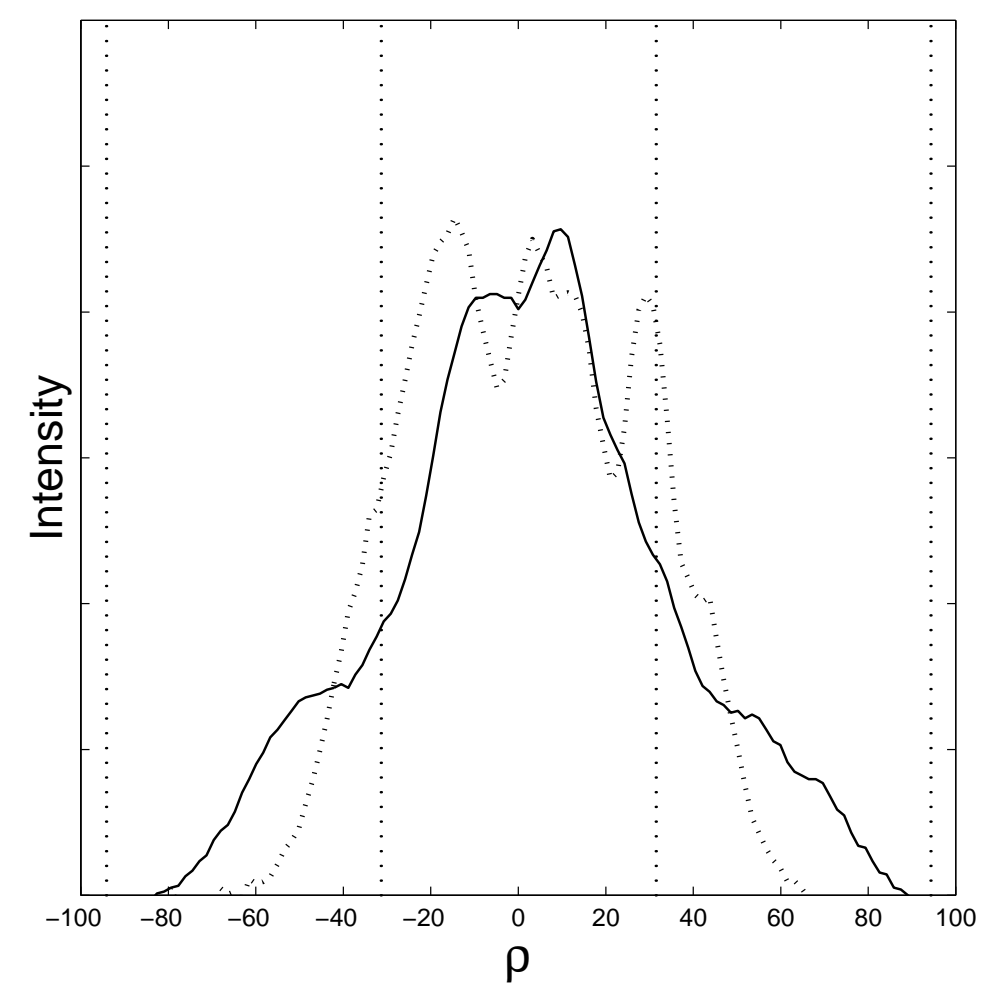

Figure 8: An example of a measured momentum distribution for kick strength $k=300$ and number of kicks $N=50$ for the double (solid line) and single (dotted line) kicked systems at a detuning of $2.8 \mathrm{GHz}$. The vertical lines correspond to the location of the barriers at $\rho= \pm 10 \pi$ and $\rho= \pm 30 \pi$. 


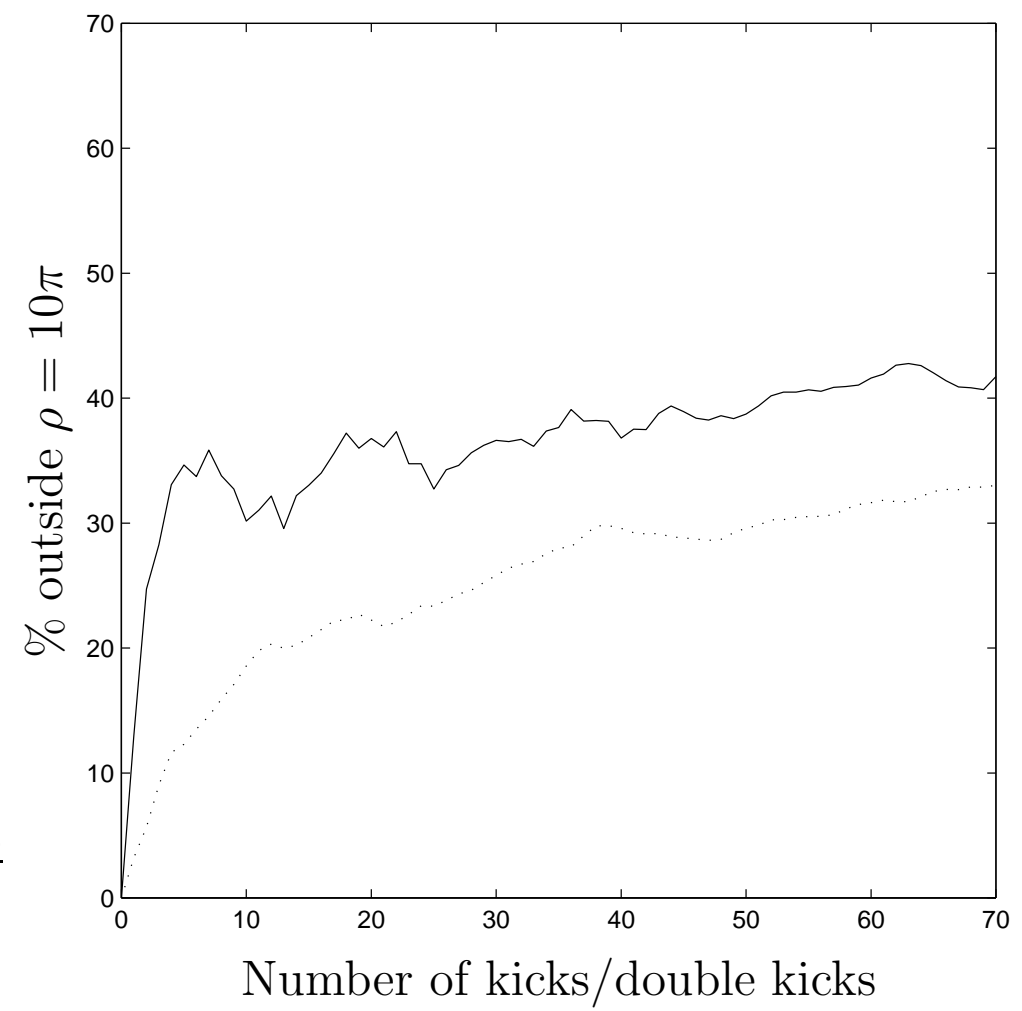

Figure 9: The calculated probability for finding particles in excess of $\rho=$ $\pm 10 \pi$, versus kick number, as calculated for a kick strength of $k=300$ and spontaneous emission rate of $\eta=0.02$ per pulse period. The solid line is the single kick, while the dotted line is the double kick. 